\title{
IoT Based Low Cost and Ageing Healthcare Monitoring System
}

\author{
K. Navya Sree, G. Mallikharjuna Rao
}

\begin{abstract}
The world's older population needs social care, healthy life, and assistive living. The health consciousness, persons care are major challenges for both researchers and industry. Health monitoring systems afford alternatives to the traditional supervision of aged patients. To reduce admissions to hospital for treatment and the cost of recognized health care. To enable disease prevention and associated lifestyle changes. Therefore, the technology has to play a key role to achieve the aspiration. In order to achieve the real-time intensive care challenge of an individual's health, early interventions and identifying the health conditions are required. In this paperwork, it is proposed to have the knowledge as soon as it causes a heart attack, it is the first step to prevent heart attack or stroke. Usually the fixed health monitoring systems are available only when the patient is lying on the bed in the hospital. This project showcases the implementation of the heartbeat, respiration, blood pressure check, pulse rate, and temperature sensing interfaced to ESP8266. This system development is for the patient at home need to be monitored by family doctor in emergency. Here, the real time patient health information is updated onto the cloud continuously. The data is for further analysis and to monitor the status of health information is retrieved to home devices and to a family doctor in critical conditions. Thus, the system can run effectively and brings a cost-effective design and implementation of data acquisition and manipulation.
\end{abstract}

Keywords: Internet of Things, ECG, Respiration, biomedical, blood pressure.

\section{INTRODUCTION}

$\mathrm{T}$ echnology is becoming essential in our daily life and it is influencing the way we work. Internet of things playing a key role to manage and control our daily life routines. The treatment of medical health data imposes stringent requirements on the system. Health is one of the global difficulties for mankind. In the last 10 years, healthcare has given a prominent role. The primary aim was to develop a system for monitoring patients by health experts, who are either in need or accomplishing their everyday life. Recently, patient health monitoring systems [1] became one of the advanced because of its upgraded technology.

Revised Manuscript Received on July 10, 2020

* Correspondence Author

K. Navya Sree*, M. Tech, CBIT, Osmania University Hyderabad, India. E-mail: navyasree.kns@gmail.com

G. Mallikharjuna Rao, Department of ECE, Osmania University, Hyderabad, India. E-mail: mallikharjunag@gamil.com

(C) The Authors. Published by Blue Eyes Intelligence Engineering and Sciences Publication (BEIESP). This is an open access article under the CC BY-NC-ND license (http://creativecommons.org/licenses/by-nc-nd/4.0/)
Nowadays, there is a need for a technologies approach. Among these traditional approaches, healthcare experts play a prominent role [2]. They need to visit the patients for necessary examination and advise. There are two problems related to this approach. Firstly, the healthcare experts must be available at the patient always and secondly, the patient should be admitted to a hospital, all the medical instructions should be followed from time to time [3,4]. To solve these problems patients should be given knowledge and information about disease identification and avoidance. Then a reliable and easily available patient health monitoring system is needed. So, we need to use advanced technology to develop the above-needed system. In recent years, health care sensors along with controller play an important role. Modern health care system introduces new technologies like wearable devices parameters which will monitor physical parameters. We can use different sensors that are available in the market now a day's such as pulse sensors, temperature sensors, pressure sensors, etc. The cost of the sensors varies depending on their size, flexibility, and accuracy.

The parameters monitored in the system are Heartbeat, Temperature, Blood Pressure and Respiration of a patient. Heartbeat is also called Pulse. Pulse is the number of times human heart beats per minute. Normally a heartbeat varies from person to person's heart in beats per minute. The average temperature of the Human body is $98.6^{\circ}$ Fahrenheit, but it may slightly vary in the range of 97.8 degrees to 99.1 degrees Fahrenheit or even may a bit higher.

The amount of the blood force against the walls of artery is Blood pressure. Blood pressure readings are expressed as a ratio of two numbers in millimeters of mercury $(\mathrm{mm} \mathrm{Hg})$. The systolic gives the pressure in the artery, when the heart beats and pushes blood in the circulatory system. The diastolic gives the pressure in the artery when the heart rests between heart beats.

The respiratory rate is given by the counting number of breaths taken per minute. When a person inhales, then oxygen enters lungs and passages to the organs. If exhale, carbon diox-ide leaves the body. A normal breathing rate plays a key role in balancing of oxygen and carbon dioxide correctly. The threshold levels with respect to the above discussed parameters are shown in the Table - I.

The Internet of Things (IoT) is the associated network between portable devices [5-7], wearable devices and house-held devices, with the controllers to the cloud servers and transfers data over a net without any necessity of human to human or human to computer interaction for the purpose of the communication between patient and the doctor.

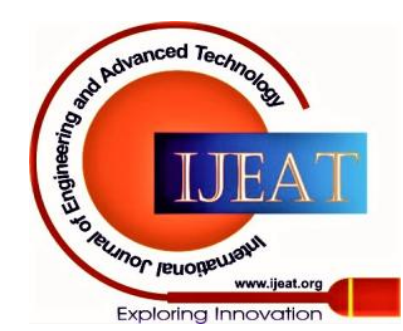


The Internet of things are playing a key role to manage and control the biomedical Sciences. The domain challenges our health and social care, overcome these issues combining social life and health with the latest technology. Therefore, technology providing services and product-based significance, to enhance the quality of living. Research areas and technology providing feasibility to solve numerous solutions of health.

The domain technology is exploited to increase the assistive techniques within the household. The ambient intelligence (AAL) make support to the people to achieve daily routines. AML is the integrity of the Technologies and the domains of computer science, engineering, and medicine. The functional ability to identify human actions from the sensors data. AAL techniques in IoT is a format that helps to communicate several devices, system growth, and task operations of a health monitoring system. There is a Rapid demand for wearables like biometric sensors and fitness trackers, where they identify the changes and analyze the sensed data $[8,9]$.

Table I: Notation of Sensors Readings

\begin{tabular}{|l|l|}
\hline \multicolumn{1}{|c|}{ Sensor } & \multicolumn{1}{c|}{ Threshold level } \\
\hline Heart rate & Less than 50 and greater than 80 \\
\hline Temperature & $\begin{array}{l}\text { Less than } 98 \text { and greater than hundred } \\
\text { in fahrenheit }\end{array}$ \\
\hline Respiration & Less than 12 and greater than 30 \\
\hline Upper blood pressure & Less than 120 and greater than 180 \\
\hline Lower blood pressure & Less than 80 and greater than 110 \\
\hline
\end{tabular}

IoT brings a direct impact on monitoring daily routine steps including diet consumed on a particular day. Smartphones are used to monitor the health and fitness based on electrocardiogram used to assess heart rate and its condition [10]. Therefore, sensors technology and the internet of things brings greater potential to improve the lifestyle. In order to provide Health Care, monitoring systems are Motto to track and control of fitness and health. Further such real-time features enable us to transmit and analyze healthcare by providing an identity of ill health symptoms. The health efficiency and cost-effectiveness are the major challenge of existing society. As per the aged population, it is significant to have advancement in Medicare for elderly people [11,12] is on-demand.

In many countries, the public health concern of aged people became costly because of raise in number and quality of service doesn't meet the societal needs. Here ok, the remote health monitoring real-time system will help us to provide solutions to overcome the challenges. Hence, continuous health monitoring of aged people using wearables and crackers provides Early Intervention Practices (EIP). According to the survey undergone in the United States and Europe identity states the patients are preferring to healthcare monitoring at home instead of going to a hospital. There are several advantages to monitor patients remotely (EIP) reducing cost and increase convenience [13].

Even health organization and the government there spending millions of dollars to provide quality of service in health sector [12].

To monitor the patient's blood pressure, temperature, and Beats Per Minute are the major concepts of health services [14]. Due to inadequate care outside the hospitals for a periodical check, accurate reports, and health records for doctors in the clinical check [15]. Therefore, combining different smart Technologies using phone, wearables, sensors and monitoring access remotely.

\section{RELATED WORK}

The research trends to advance the technology in a broad way of AAL platforms helps to assist aging adults in the residence. The non-invasive devices are used to monitor through wearable sensors. The crucial development of facilities to monitor health status independently at home or at work. Both data acquisition and data processing of real-time sensor data is done outside the hospital [16].

Researchers and industry projects related to the AAL area [17] play a vital role to monitoring and save lives by altering in Prior. Monitoring helps to diagnose the heart problems, heart-related projects associated with Philips [18,19].

A group of projects which are incorporated with aged people at home. An assisted living joint program funded for these types of projects. This project $[20,21]$ includes sensors to monitor and help adults. The home project is a process of monitoring [22] residents through a bot. In some projects, it is focused to monitor patients suffering from the chronic disease, such as CASAS project [23]. A telehealth Lifestyle system is based on the monitoring of information gadgets from the patients. Projects like [24] biomedical, which collects the electrocardiogram data. The main aim is to detect the illness earlier and then assess healthcare.

The researchers and industry are looking forward to automating the Healthcare products [25] in a more convenient way. Remote health monitoring systems are increasing due to the cost of healthcare [12] and rapid increase of ageing population. Therefore, solving healthcare issues becoming a growing field. After investigating several researchers. Experimenting Smart Home healthcare environment on cloud (CoSHIE). The non-invasive sensors gather the information from the the the patient, according to its daily activities. The effective collection of behavioral changes from the sensors and forward it to the cloud via home gateway. CoSHIE is hybrid data storage, which includes a non-relational and relational data center. The author has selected data is stored in the database, in terms of name, sex, fluid intake, and Spo2.

The boxer based on Raspberry Pi and Arduino for remote monitoring Healthcare by [14], access the ECG. Hear the Raspberry pi is used as a gateway to collect the ECG data from wearable and store it in the database. GSM is used to send an alert to a doctor in an emergency. The data is available to the authorized person through a web app. Wireless sensor networks are used to collect the blood pressure coma heart rate and ECG [26,27]. As mentioned by the author the system is capable to provide patient information to the family doctor in an emergency. Arduino is used as a gateway to communicate the patient's information in an emergency to the doctor [15].

\section{PROPOSED SYSTEM}

The system is to help the chronic people elderly persons with their health conditions without hospitalization. The major approaches to enable the integrity of the assistant and management and self-monitoring. In order to perform these four major features of the system for data acquisition in terms of temperature, blood pressure, respiration, and ECG. To conduct an experiment, 
we need a setup where it can provide accurate clinical setup and can be used for the general purpose. The wearable sensors and network services are required, to prepare the infrastructure of this technology.

\section{A.System architecture}

The basic needs of the system architecture are sensors, computing device, Wi-Fi, in Nodemcu or through mobile. The testing equipment consists of user interface and internet connectivity. The user interfaces as Nodemcu with wearables for sensing heartbeat, temperature, blood pressure, and respiration. The Healthcare system makes convenience to the patient for monitoring his health at home. The system is built with a three-layer portion, that needs the integrity to conclude an approach. The basic requirements are wearable sensors integrated to gateway Cloud Service data centre to store and process the data. Therefore, the layers are as shown in Fig. 1.

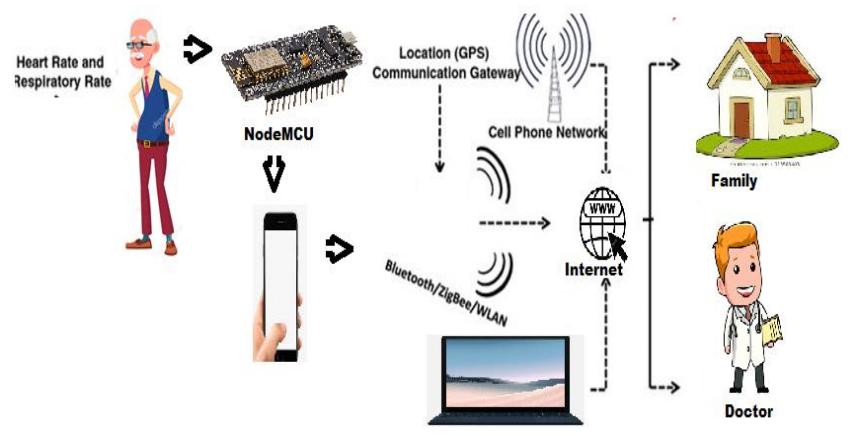

Fig. 1. System architecture of health monitoring system

\section{B. Wearable devices}

The nodemcu with sensors will make a physical existence of sensors sensing the data from the patient's body to collect the psychological status. The sensors such as heart rate in beats per minute, the temperature of the human body in degree centigrade blood pressure variability, and respiration rate in breaths per minute $[28,29]$. Therefore, monitoring these sentences patients' body in an abnormal state. The system helps the patient to monitor continuously for the abnormality of the patient's body detected by the sensors [30]. The abnormality is due to the oxygen levels in a body degrades to sleep apnea, it may lead to the death of a person. If high blood pressure occurs it may lead to diabetes or kidney failure. Therefore, the sensed data of the patient is transmitted to the Smartphone via Bluetooth is communicated to the cloud database. Continuous measures of the patient data are updated periodically using internet of things. Hence, it helps to improve the comfortability of the patient and to enhance the quality of service.

\section{Cloud computation}

. Instead of having personal devices to store the data or depending upon computing devices shared for local applications. The services relied on the internet, exclusively for data storage, for the improved computing power. The patient's data is transmitted through Wi-Fi over the internet to the Stored database. In case of emergency, this data becomes available for further investigation by the doctor. The complete data analysis is performed in the cloud and processing of disorder detection in the patient's data. To check their health changes in patients' data are categorized based on the disease and patient health condition. The cloud can be used exclusively for Analytics, and to allow the medical professionals to diagnosis the patient status. It becomes easy for the doctors to prescribe the medicine immediately, according to the real-time updates of the patient in absence.

\section{Monitoring platform}

As the sensory data is uploaded into the cloud the doctors have a platform, where they can access the patient records. In such a case, the doctors are capable enough to inspect the reports stored in the cloud and possible to take necessary actions.

\section{SOFTWARE AND APPS}

The Arduino IDE software is for writing a program in C and uploading to NodeMCU Board. NodeMCU will send the information through the server using Wi-Fi module to cloud and mobile applications. The cloud application used here is the ThingSpeak App and mobile application used is Blynk App.

\section{A.ThingSpeak App}

We have used mobile and cloud server applications to save and display the data obtained from the sensors transferred to micro-controller. The ThingSpeak App which displays the sensed data obtained in the form of graphs as shown in Fig. 2. The different channels are used for different graphs representation. ThingSpeak is a source of IoT application that displays the data and even it can store and retrieve, when required in future.

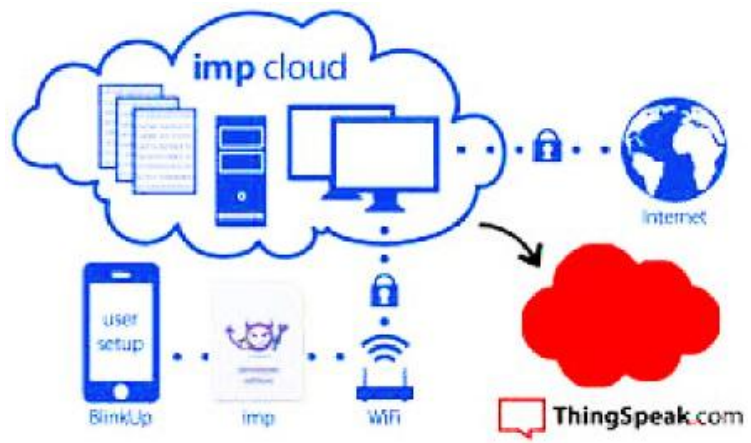

Fig. 1. ThingSpeak App process

\section{B.Blynk App}

The mobile application used here is Blynk App displays the data obtained from the sensors, through micro-controller. This application will even store data, visualize it and can use it for future use. Using this application, we can monitor a patient from anywhere.

In this application various LCD Displays, charts, gauges as shown in Fig. 3 used for displaying the sensed real-time data presents the health constraints of a patient. Through this application doctor or any person can monitor a person's health conditions from anywhere they are. As a future scope of project call can also be done in any emergency conditions from the parameters obtained which are sensed. The applications were connected to an IoT cloud and figured the real time data by an LCD display.

Published By:

Blue Eyes Intelligence Engineering

\& Sciences Publication

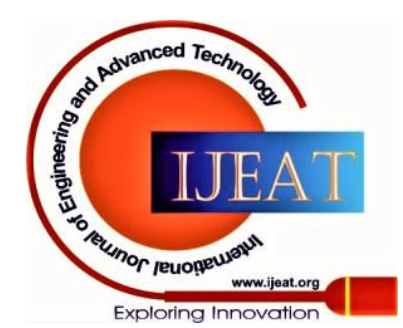




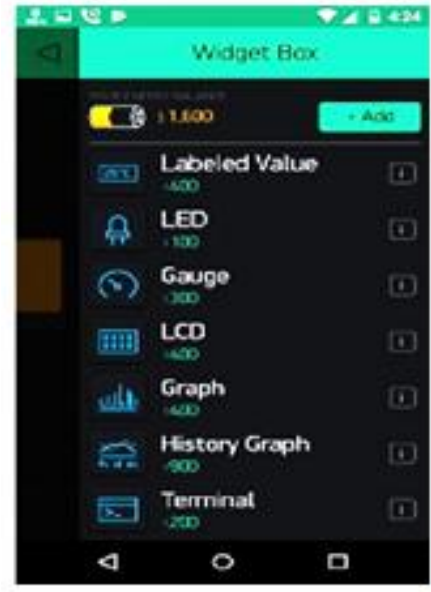

Fig. 3. Different types of charts to display for data obtained

\section{DESIGN AND IMPLEMENTATION}

The health monitoring system is designed for automatic systems. It is unable to send the real-time data and monitor, in accordance with the environmental conditions. The data is sent wirelessly through Wi-Fi and Bluetooth for the smartphone as a gateway. The data analysis brings is services provided by the doctor through apps.

\section{A.Components Required}

The major components that are required for building a project is discussed in a detailed give matter solution. The medical applications are a critical job to handle the data through components that are used for the purpose. Therefore, the list of components is listed to optimize the cost, accuracy, and robots are the products used in the development of the product. The listed components given is an accurate result as they are proposed in the design and implementation in the project are given in the Table- II.

Table- II: Components List required for the project

\begin{tabular}{|l|l|}
\hline \multicolumn{1}{|c|}{ Name of the components } & \multicolumn{1}{c|}{ Purpose } \\
\hline ESP 8266 & Wi-Fi module \\
\hline Heart rate sensor ( pulse sensor) & Heartbeat measurement \\
\hline Temperature sensor (Ds18b20) & $\begin{array}{l}\text { Measure the body } \\
\text { temperature }\end{array}$ \\
\hline Blood pressure sensor (MPX42500) & Measure the blood pressure \\
\hline Respiration sensor & Measure respiration \\
\hline Adafruit & Convert analogue to digital \\
\hline
\end{tabular}

\section{B. Node MCU ESP8266}

NodeMCU is an open-source LUA form firmware advanced for ESP8266 Wi-Fi chip as in Fig. 4. It is consisting of 30 pins and it has Analog and Digital pins. The serial communication protocols are UART, SPI, and I2C available in NodeMCU.

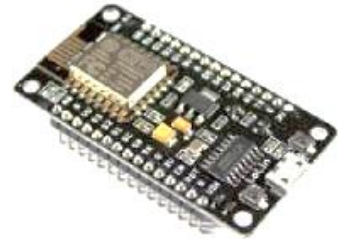

Fig. 4. NodeMCU ESP8266

\section{C.Heart Rate Sensor}

The pulse sensor shown in Fig. 5 is used to sense the Heart beat of a patient. It is interfaced with NodeMCU by connecting
VCC, GND and A0 pins. It gives the pulses for every minute called beats per minute. Its operating voltage is $+5 \mathrm{~V}$ to $+3.3 \mathrm{~V}$.

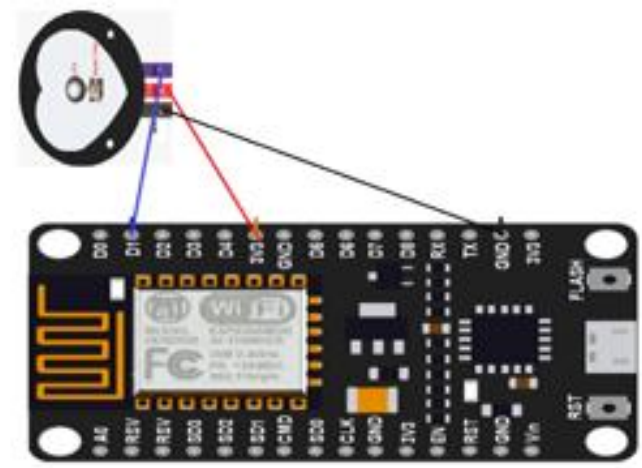

Fig. 5. Pulse sensor interface

\section{D.Temperature Sensor}

The temperature sensor used is the Ds18b20 sensor as shown in Fig. 6. It is a waterproof sensor. The power supply range is $3.0 . \mathrm{V}$ to $5.5 \mathrm{~V}$. The temperature obtained is in centigrade and then it is converted to Fahrenheit through the software code.

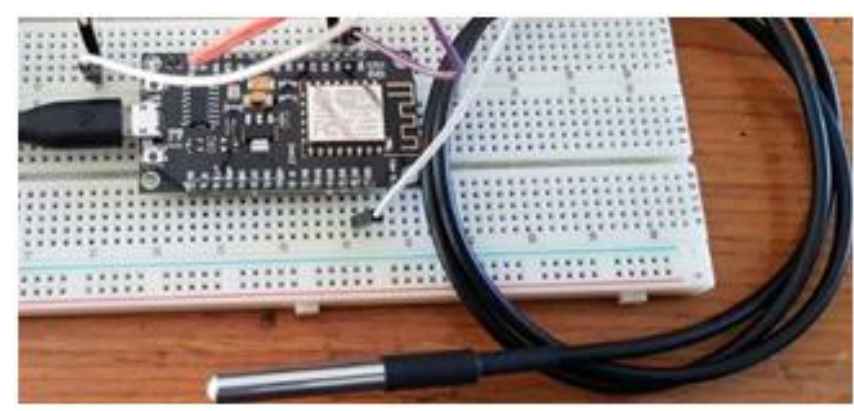

Fig. 6. Ds18b20 Temperature sensor interface

\section{E.Blood Pressure Sensor}

MPX4250 Fig. 7 series piezo resistant transducer is a monolithic pressure sensor made of silicon used in extensive range of applications, mainly those using a microcontroller or microprocessor with Analog and Digital inputs. It is a analog output signal that is related to applied pressure.

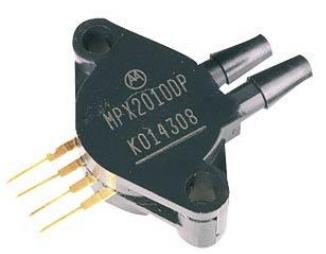

Fig. 7. MPX42500 Blood pressure sensor inteface

\section{F. Respiration Sensor}

The respiration sensor used in this system is a sound sensor or microphone sensor as in Fig. 8. The sound sensor works on the basis of the microphone which detects sound. It gives the measurement of how loud the sound is. The module used as a microphone sensor is LM393.

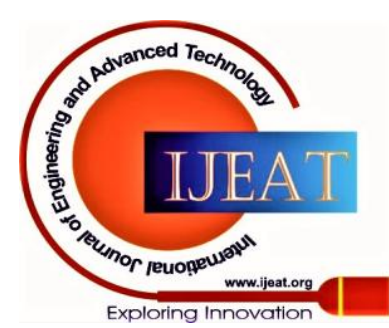




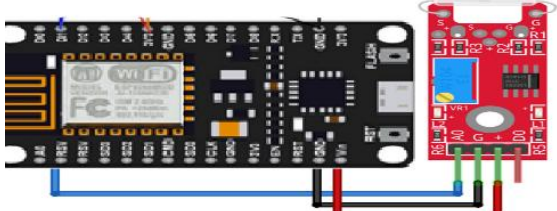

Fig. 8. LM393 Sound Sensor interface

\section{G. Ads1115 Adafruit I2c}

The micro-controllers lacking an analog to digital converter or for a high precision ADC, the ADS1115 as shown in Fig. 9, enables 16-bit precision at 860 samples/ second in I2C. The chip is configured as 4 single-ended input channels or two differential channels.

The ThingSpeak IoT cloud platform is used as an IoT cloud and Blynk App as the mobile application for proposed system. The total system block diagram is shown in Fig. 10. The condition of the patient is determined.

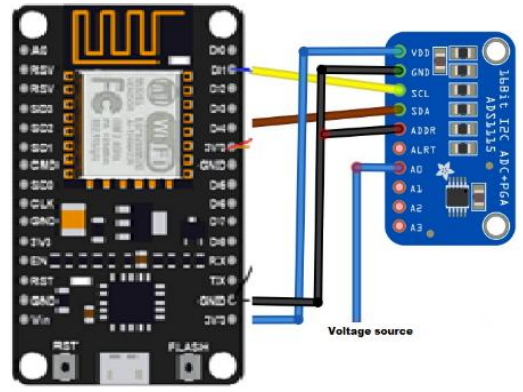

Fig. 9. ADS1115 Adafruit interface to NodeMCU

\section{H. System Implementation}

We have used NodeMCU (IoT development board) with Wi-Fi module ESP8266 as a principal controller of our proposed health monitoring system. The NodeMCU will collect the information of a patient's health constraints for the various sensors described in Table- I, which are directly connected with the principle. The system implementation setup is shown in the above Fig. 10. shows the connections of the NodeMCU ESP8266 and the sensors. The complete hardware setup is as shown in Fig. 11 to perform the four major operations and have a collective information of a patient.

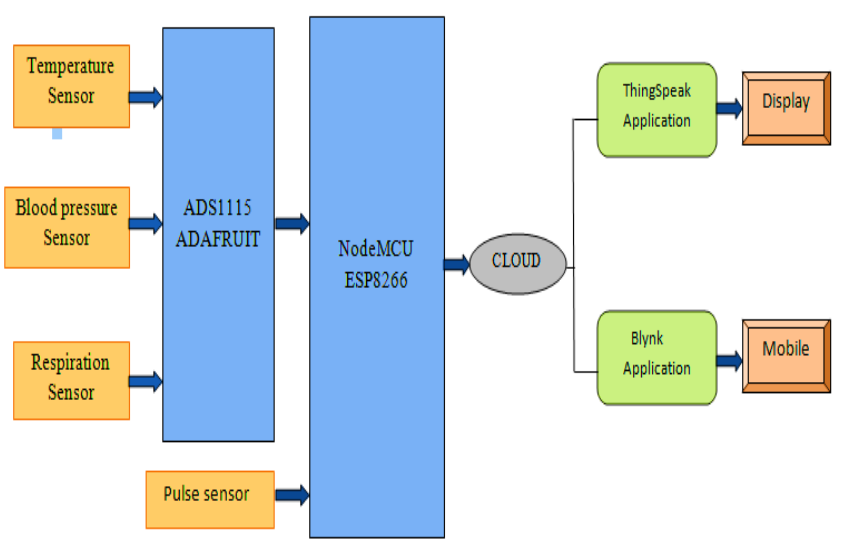

Fig. 10. Block Diagram of the proposed Patient Health monitoring system

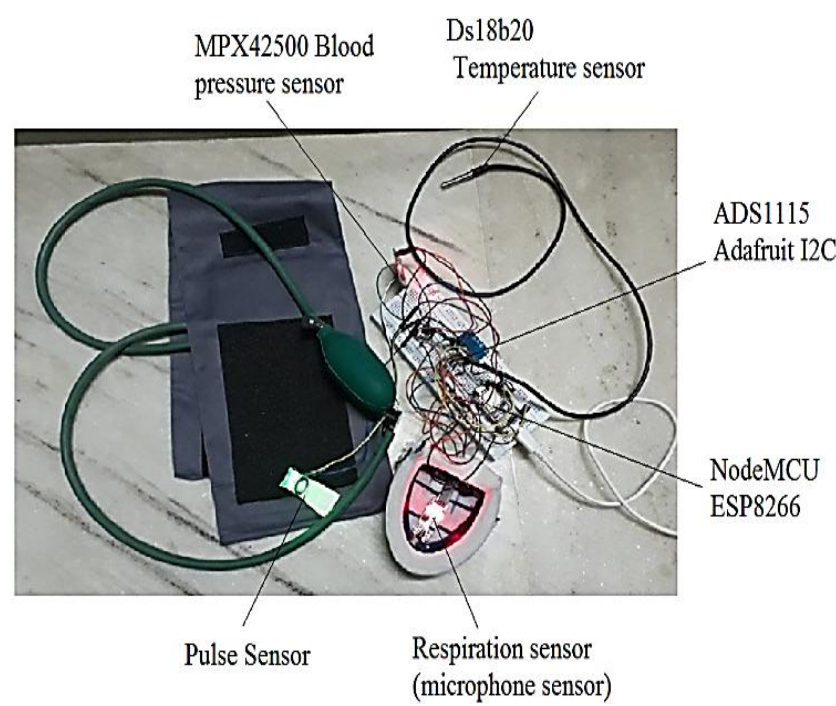

Fig. 11. Complete wearabele setup of health monitoring system

\section{RESULTS OBTAINED}

The data obtained from the wearable sensors through NodeMCU ESP8266 is of a middle-aged person, which is displayed through a server in Thing Speak App and mobile through Blynk App.

\section{A. Heartbeat Rate In Bpm (Beats Per Minute)}

The implementation results obtained from the Heartbeat of a middle-aged person from the pulse sensor are given below.

1) Outputs obtained in ThingSpeak App: The heart-beat obtained in the ThingSpeak app which is the first field Fig. 12 of the channel with database taken from the cloud. It is within the range of 72 to 95 BPM's which are taken across the timing slot 12:10:20 and even at 12:10:40 which is the normal heart rate of a middle-aged person. The obtained data will be stored in the channel.

2) Outputs Obtained in Blynk App: The heart-beat values shown in Fig. 13 are obtained from the sensor are displayed in Blynk app. Where a, b is the heart rates taken at certain time slots which are the normal Heartbeat of middle-aged person.

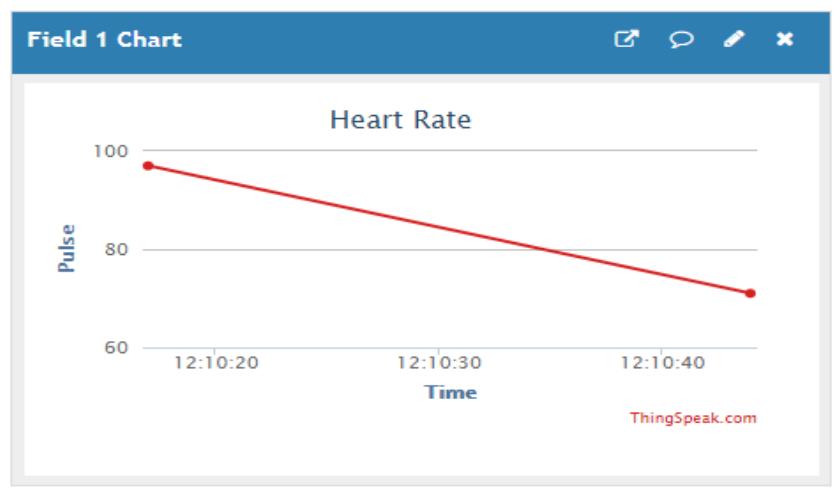

Fig. 12. The Heart rate obtained in the ThingSpeak App

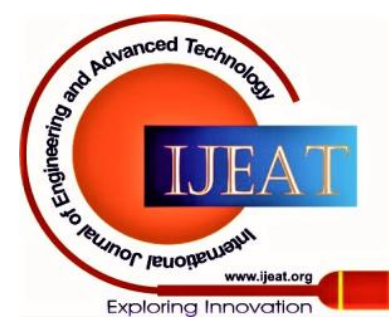




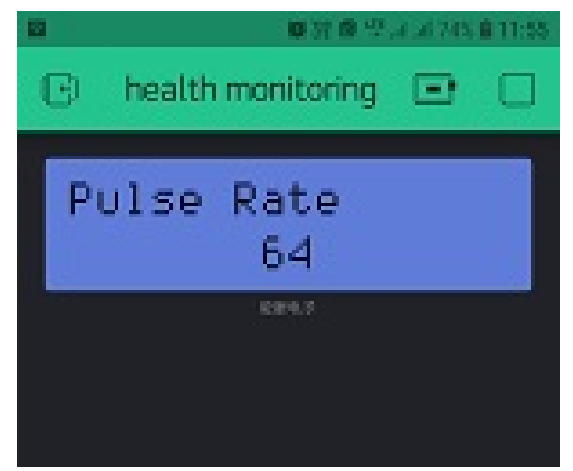

Fig. 13. The Heart rate obtained in the Blynk software

Sensing the heart rate variability is the major information that is required to understand the concept of sensitivity. Where it will synchronize with the blood flow. The pulse sensor circuit consists of an LED and a photodiode. The LED exhibits light when a finger is placed on the cover of the pulse sensor, the reflection of a signal is detected by a photodiode. Therefore, to calculate the beats per minute the peak values of PQRST of heart variability enable Heartbeat ECG access. The normal beat consists of the P-wave, QRS-segment, and ST-segment. The P-waves indicate the electrical activity of the artificial depolarization of a small chamber of the heart.

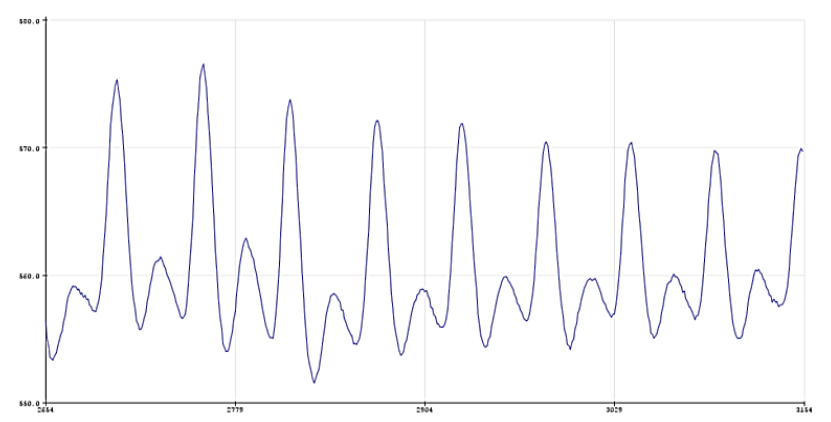

Fig. 14. Observation of heart rate variability

Table - II Maximum heart rate with respect to Age

\begin{tabular}{|c|c|c|}
\hline Age & $\begin{array}{l}\text { Estimated } \\
\text { maximum } \\
\text { heart rate }\end{array}$ & $\begin{array}{l}\text { Target heart } \\
\text { rate (60-85 } \\
\text { percent of } \\
\text { max) }\end{array}$ \\
\hline 20 & 200 & $120-170$ \\
\hline 25 & 195 & $117-166$ \\
\hline 30 & 190 & $114-162$ \\
\hline 35 & 185 & $111-157$ \\
\hline 40 & 180 & $108-153$ \\
\hline 45 & 175 & $105-149$ \\
\hline 50 & 170 & $102-145$ \\
\hline 55 & 165 & $99-140$ \\
\hline 60 & 160 & $96-136$ \\
\hline 65 & 155 & $93-132$ \\
\hline 70 & 150 & $90-123$ \\
\hline
\end{tabular}

The QRS complex is the visible portion of the ECG as shown in the figure. It is in the form of triangle and easy to identify the shape in the waveform. It is the electrical activity of the ventricular depolarization of large Chambers of heart. The ST-segment is after the QRS-complex, it is a flat area of the next periodic shape of ECG. It provides crucial information on heart attacks. Therefore, counting the spaces between QRS Complex within 6 seconds is considered for the calculation of BPM. The number of beats is measured in one minute. The witness and the age, based on temperature emotional status, and typhoid conditions will impact on the heartbeat. The typical heart rate varibility is given in Table -II.

The number of times the heart beats in a minute is termed as heart rate. It depends upon the existence of your body, under stress, due to exercise, dizziness, and general Health. The age and fitness impact the heart rate may also influence the parameters due to body temperature, emotional status, medication, and heart conditions. Don't try to check the pulse rate on Thumb as the artery becomes harder to count at ridley. Count the number of pulses in 15 seconds then multiply the number by 4 gives the actual heart rate.

\section{B. Temperature Of Human Body}

The implementation results obtained from the Ds18b20 sensor are the temperature of a healthy middle-aged person which is plotted below.

1) Outputs obtained in ThingSpeak App: The obtained temperature sensor values displayed in ThinkSpeak Fig. 15 at the 2nd field as a waveform which is constant with the value of 97.5F. The temperature data are taken from the cloud with time slots of 5 secs each.

2) Outputs obtained in Blynk App: The Temperature of the Human body Fig. 16 obtained from the temperature. sensor sends through the cloud to the Blynk App is displayed using LCD. The temperature displayed is in both centigrade and Fahrenheit scale which is a healthy middle-aged person reading.

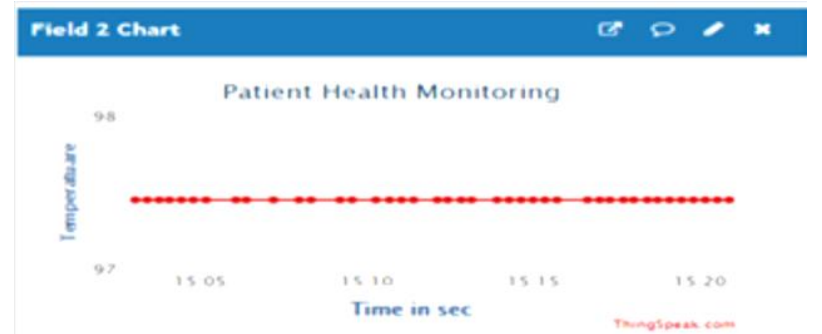

Fig. 15. The temperature obtained in the ThingSpeak App

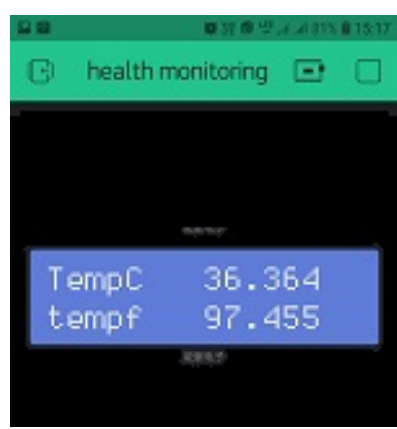

Fig. 16. Temperature in Fahrenheit obtained in Blynk software

\section{C.Blood Pressure}

The implementation results obtained from the MPX42500 pressure sensor are the reading of healthy middle-aged persons plotted below.

\section{Published By:}

Blue Eyes Intelligence Engineering

\& Sciences Publication

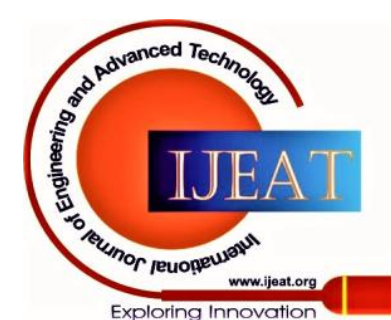


1) Outputs obtained in ThingSpeak App: The blood pressure data in Fig. 17 are obtained from the sensor taken from the cloud. These obtained results are charted in ThingSpeak in Field 3 where there occurred a constant blood pressure of 1.45 at time slots of 15 secs.

2) Outputs obtained in Blynk App: The Blood pressure obtained is the readings of a healthy middle-aged person taken through the cloud and the value is constant at 1.45 is a ratio of systolic and diastolic values displayed in Blynk app Fig. 18.

Two different readings are considered to record blood pressure. The readings are systolic and diastolic. For example, the blood pressure values recorded as $120 / 80$ $\mathrm{mmHg}$ (millimeters of Mercury). Where 120 is the systolic pressure and 80 is the diastolic pressure. The contraction of heart artery to pump blood used to measure systolic pressure. Resting of the heart artery between the beats is the diastolic pressure. The higher the values of recordings of the heart, states that the heart is working hard to pump the blood in the arteries. It may cause narrow blood vessels stressed and scared. Preferable doctors supposed to check the blood pressure before and after medication, at certain periods in a day, dizzy or stressed.

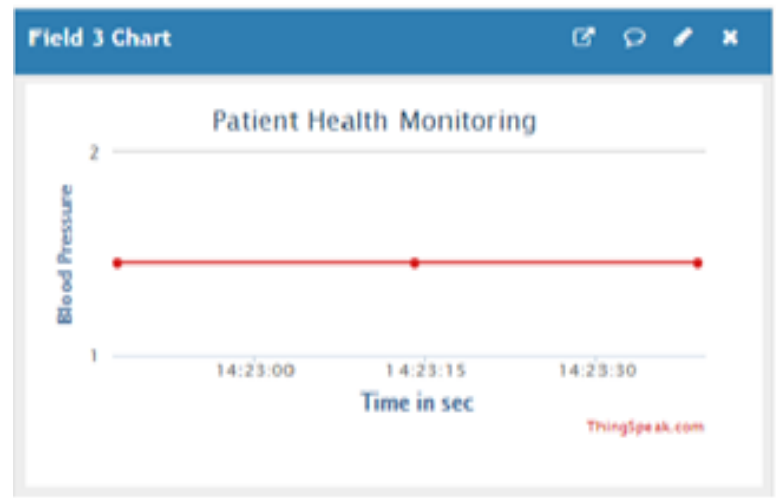

Fig. 17. Blood pressure obtained in Blynk software

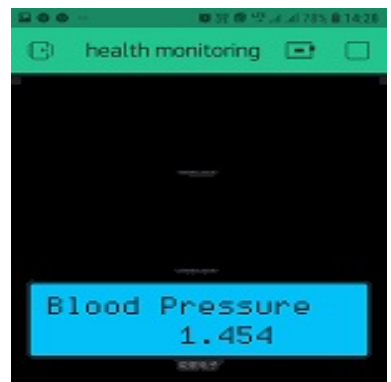

Fig. 18. Blood pressure obtained in ThingSpeak App

\section{D.Respiration Rate In Bpm(Breaths Per Minute)}

The implementation results obtained from the sound sensor are healthy middle-aged person readings given below. 1) Outputs obtained in ThingSpeak App: The respiration rate obtained from the sensors taken from the cloud and plotted in ThinkSpeak in the field 4 in a channel as shown in Fig. 19. The respiration plot obtained consists of values plotted for every 5 secs. The average values of the BPM's obtained are 16 BPM which is a healthy middle-aged person respiration rate.

2) Outputs obtained in Blynk App: The respiration rate obtained from the sensor is taken from the cloud and displayed in Blynk with pin number V4 as shown in Fig. 20 The respiration rates obtained are reading of a healthy middle-aged person which can be operated from anywhere.

\section{Table - III: Measures for systolic and diastolic of Blood Pressure}

\begin{tabular}{|l|l|l|}
\hline \multicolumn{1}{|c|}{ Category } & \multicolumn{1}{c|}{ Systolic } & \multicolumn{1}{c|}{ Diastolic } \\
\hline Normal & less than 120 & and less than 80 \\
\hline Elevated & $120-129$ & and less than 80 \\
\hline $\begin{array}{l}\text { High blood pressure } \\
\text { stage 1 (hypertension) }\end{array}$ & $130-139$ & or 80-89 \\
\hline $\begin{array}{l}\text { High blood pressure } \\
\text { stage 2 (hypertension) }\end{array}$ & 140 or higher & or 90 or higher \\
\hline $\begin{array}{l}\text { hypertensive crisis (call } \\
\text { your local emergency } \\
\text { services) }\end{array}$ & higher than 180 & higher than 120 \\
\hline
\end{tabular}

Fig. 19. The respiration rate obtained in Blynk App

The respiratory system of a person measured in terms of number of breaths per minute. The normal rate in the adults at rest will vary from 12 to 20 breaths per minute. In the abnormal states like penumonia, drug overdose, anxiety and asthma that can impact the respiratory system as given in Table - III.

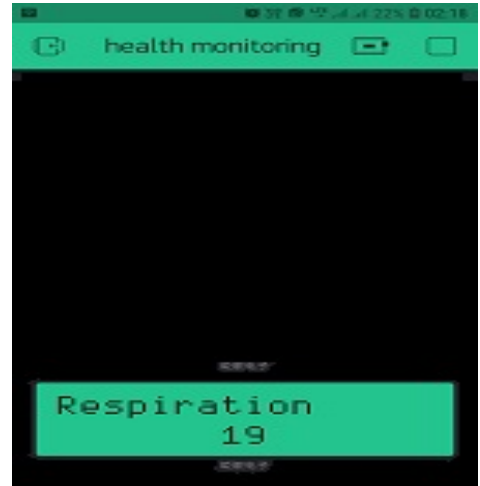

Fig. 20. The respiration rate obtained in ThingSpeak App

\section{E.Patient Health Monitoring System}

The outputs plotted in Fig. 21 from pulse sensor, temperature sensor, blood pressure sensor, and respiration sensor are taken from the cloud and attached with the ThingSpeak with the auth token in fields:1,2,3,4 in a channel. the output waveform obtained are plotted.

The output obtained in Fig. 22 by all the sensors in the patient health monitoring are considered and taken from the cloud which is connected with the pins V1, V2, V3, and V4. The patient health monitoring outputs obtained in the Blynk App are shown below.

The data is sent to cloud, where ThingSpeak monitors the patient information in the form of graphs. The data getting from the cloud is even displayed in mobile using the Blynk App which is more portable to the user and a doctor.

Published By:

Blue Eyes Intelligence Engineering

\& Sciences Publication

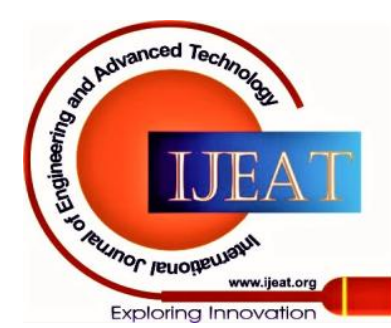




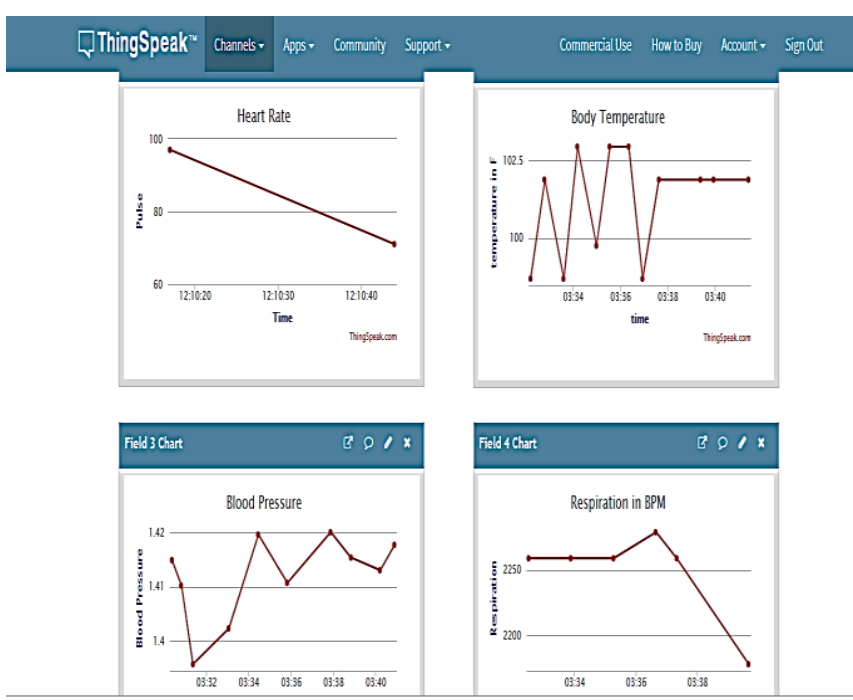

Fig. 21. Patient Health monitoring system outputs obtained in ThingSpeak app

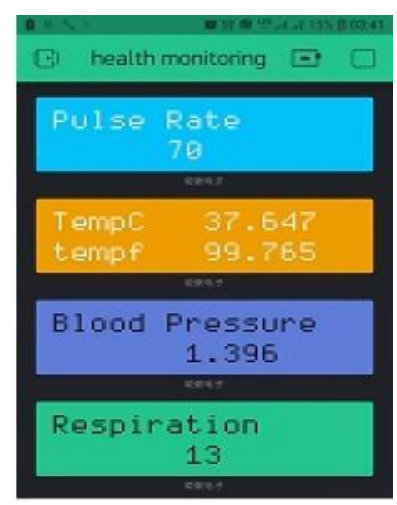

Fig. 22. Patient health monitoring results in Blynk App

\section{CONCLUSION}

The ageing healthcare monitoring system is a challenging approach that will provide healthcare for older people to avoid hospitalization. There is much demand for effective health care product solutions to monitor older people at home in real time. The system helps us to provide convenience, safe social care for disabled chronic, and aged people. The continuous real-time monitoring remotely, will provide liveliness of healthcare. In emergencies or in critical situation of patients' health information, is reported to the family doctor. The hardware interfacing module is implemented using ESP8266, which is a wireless-based health monitoring system to sense heartbeat, body temperature, Blood pressure, and Respiration. The sensed information is sent to the cloud wirelessly. The hardware module helps in real-time health monitoring systems at home gives the information and provides quick service if needed. Thus, the overall system can be optimized with ultra-low power consumption, ease of use, simple to implement, cost effective and portable to the user. The system may be helpful to monitor the patient remotely but may not be suitable for clinical purpose.

\section{REFERENCES}

1. Mohammed Al-khafajiy, Thar Baker, Carl Chalmers,"Remote health monitoring of elderly through wearable sensors", Multimedia Tools and Applications, pp. 24681 - 24706, 2019.

2. S Pradeep Kumar, Vemuri Richard Ranjan Samson, U Bharath Sai,
"Smart Health Monitoring System of Patient Through IoT" International conference on I-SMAC, pp. 551-556,2017.

3. Veena Tripathi, Faizan Shakeel, "Monitoring Health Care System using Internet of Things - An Immaculate Pairing", International Conference on Next Generation Computing and Information Systems (ICNGCIS), pp.153- 158, 2017.

4. Nawras GEORGI, Regine ' LE BOUQUIN JEANNES,' Proposal of a Remote Monitoring System for Elderly Health Prevention" International Conference on Smart, Monitored and Controlled Cities (SM2C), pp.69-74, 2017

5. Jorge Gómeza, Byron Oviedob, Emilio Zhumab," Patient Monitoring System Based on Internet of Things", 7th International Conference on Ambient Systems, Networks and Technologies (ANT 2016), pp. 90-97, 2016

6. Zia Uddin Ahmed, Mohammad Golam Mortuza, Mohammed Jashim Uddin, Md. Humayun Kabir, Md. Mahiuddin, MD. Jiabul Hoque," Internet of Things Based Patient Health Monitoring System Using Wearable Biomedical Device”, ICIET, 2018.

7. Neel Kamal, Prasun Ghosal, "Three Tier Architecture for IoT Driven Health Monitoring System Using Raspberry Pi", IEEE International Symposium on Smart Electronic Systems (iSES), pp.167- 170, 2018.

8. Parag Chatterjee, Leandro J. Cymberknop, Ricardo L. Armentano" IoT-based Decision Support System for Intelligent Healthcare Applied to Cardiovascular Diseases", 7th International Conference on Communication Systems and Network Technologies, pp. 362- 366, 2017.

9. Angelini L, Carrino S, Abou Khaled O, Riva-Mossman S, Mugellini E, "Senior living lab: an ecological approach to foster social innovation in an ageing society". Fut Internet, 2016.

10. Bonato P, "Wearable sensors and systems.", IEEE Eng Med Biol Mag, pp. 25-36, 2010.

11. Oresko JJ, Jin Z, Cheng J, Huang S, Sun Y, Duschl H, Cheng AC () A wearable smartphone-based platform for real-time cardiovascular disease detection via electrocardiogram processing. IEEE Trans Inf Technol Biomed, pp. 734-740, 2010.

12. Patel S, Park H, Bonato P, Chan L, Rodgers M "A review of wearable sensors and systems with application in rehabilitation", Neuroeng Rehabil, 2012.

13. Pham M, Mengistu Y, Do HM, Sheng W "Cloud-Based Smart Home Environment (CoSHE)for home healthcare" In: 2016 IEEE international conference on automation science and engineering (CASE). IEEE, pp 483-488, 2016.

14. Navarro J, Vida na-Vila E, Alsina-Pag`es RM, Herv'as M () Real-Time Distributed architecture for remote acoustic elderly monitoring in Residential-Scale ambient assisted living scenarios. Sensors (Basel Switzerland) sa, 2018.

15. Gupta MS, Patchava V, Menezes V, "Healthcare based on IoT using Raspberry Pi”, In: 2015 international conference on green computing and internet of things (ICGCIoT). IEEE, pp 796-799, 2015.

16. Dudakiya S, Galani H, Shaikh A, Thanki D, Late RA, Pawar SE" Monitoring mobile patients using predictive analysis by data from wearable sensors." In: International conference on electrical, electronics, and optimization techniques (ICEEOT). IEEE, pp 332-335, 2016

17. Palumbo F, Ullberg J, timec A, Furfari F, Karlsson L, Coradeschi S, "Sensor network infrastructure for a home care monitoring system". Sensors. 2014.

18. Luprano J, Sol’a J, Dasen S, Koller JM, Ch'etelat O, "Combination of body sensor networks and on-body signal processing algorithms: the practical case of MyHeart project.” IEEE, pp 76-79, 2016.

19. Chouvarda I, Antony R, Torabi A, Weston J, Caffarel J, van Gils M, Cleland J, Maglaveras N, "Temporal Variation in telemonitoring data: on the Effect of Medication and Lifestyle Compliance", International Journal of Bioelectromagnetism, 2013.

20. Luprano J, De Carvalho P, Eilebrecht B, Kortelainen J, Muehlsteff J, Sipila A, Sol’a J, Teichmann D, Ulbrich M "HeartCycle: advanced sensors for telehealth applications", 35th annual international conference of the IEEE engineering in medicine and biology society (EMBC), 2013.

21. Chen TL, King CH, Thomaz AL, Kemp CC "Touched by a robot: an investigation of subjective responses to robot-initiated touch" 6th ACM/IEEE international conference human-robot interaction (HRI), 2011.

22. Cook D, Das SK "Smart environments: technology, protocols and applications”, Wiley, New York. 2004.

Published By:

Blue Eyes Intelligence Engineering

\& Sciences Publication

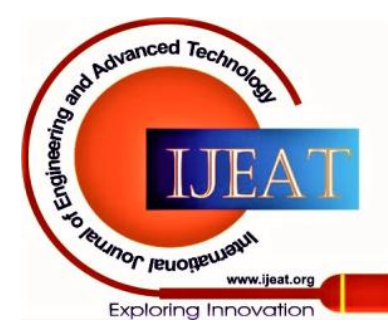


23. Yamazaki T, "The ubiquitous home”, Int J Smart Home, pp. 17-22, 2007.

24. Rashidi P, Cook DJ, "Keeping the resident in the loop: Adapting the smart home to the user", IEEE Transactions on systems, man, and cybernetics-part A: systems and humans, 2009

25. Tamura T, Kawarada A, Nambu M, Tsukada A, Sasaki K, Yamakoshi KI "E-healthcare at an experimental welfare techno house in Japan", The Open Medical Informatics Journal, 2007.

26. Islam SR, Kwak D, Kabir MH, Hossain M, Kwak KS () The internet of things for health care: a comprehensive survey. IEEE Access, pp.678-708, 2015.

27. Ali A, Ming Y, Chakraborty S, Iram S, “A comprehensive survey on real-time applications of WSN", Fut Internet, 2017.

28. Chauhan J, Bojewar, "Sensor networks-based healthcare monitoring system", In: International conference on inventive computation technologies (ICICT). IEEE, Vol 2, pp. 1-6. 2016.

29. Nienhold D, Dornberger R, Korkut S, "Sensor-Based Tracking and Big Data Processing of Patient Activities in Ambient Assisted Living", IEEE international conference on healthcare informatics (ICHI). IEEE, pp 473-482, 2016.

30. Pantelopoulos A, Bourbakis NG "A survey on wearable sensor-based systems for health monitoring and prognosis", IEEE Trans Syst Man Cybern, 2010.

\section{AUTHORS PROFILE}

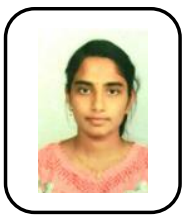

K. Navya Sree is currently pursuing Masters of Technology (MTech) under Osmania University with the specialization of Embedded Systems and VLSI Design. I had completed Bachelor of Engineering with specialization of Electronics and Communication Engineering under Jawaharlal Nehru Technology University.

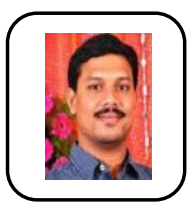

G.Mallikharjuna Rao is employed as Assistan Professor in the Electronics and Communication Engineering department and accompanying to organize student club activities and engineering research. He has guided numerous projects under his guidance. He completed his $\quad$ B. Tech degree from University of Madras. He finished his M. Tech degree from Koneru Lakshmaiah University and pursuing Ph.D from Osmania University. He published International journals and conferences. His area of speciliazation is inforamtion security.

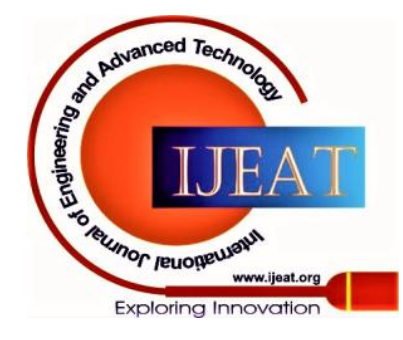

